Canad. Math. Bull. Vol. 21 (2), 1978

\title{
ON THE INPUT-OUTPUT STABILITY OF LINEAR CONTROLLABLE SYSTEMS
}

\author{
BY \\ MIHAIL MEGAN
}

1. Introduction. The extension of bounded input, bounded output criteria of Perron [1] for the case of a linear control system

$$
(A, B) \quad \dot{x}(t)=A(t) x(t)+B(t) f(t) \quad\left(\dot{x}=\frac{d x}{d t}\right)
$$

has been widely discussed (cf. e.g., references [2] to [10]).

As is well known ([2]) the equation

$$
\dot{x}(t)=A(t) x(t), \quad t \geq 0
$$

is exponentially stable iff there exist positive constant $N$ and $\nu$ such that

$$
\|U(t, s)\| \leq N \cdot e^{-\nu(t-s)}
$$

for all $s \geq 0$ and for all $t \geq s$, where $U(t, s)$ is the evolution operator of the equation $(A)$.

Throughout in this paper we shall assume that a constant $K>0$ exists such that

$$
\|A(t)\| \leq K, \quad\|B(t)\| \leq K
$$

for all $t \geq 0$.

A system satisfying (1.1) will be termed a bounded system.

Following [2], we say that the system $(A, B)$ is (zero-state) bounded-input, bounded-output stable iff for every bounded input $f$ there exists a constant $N$ such that the output $x\left(t, t_{0} ; 0 ; f\right)$ satisfies the condition

$$
\left\|x\left(t, t_{0} ; 0 ; f\right)\right\| \leq N, \text { for all } t \geq t_{0} \geq 0 .
$$

We denote that the bound $N$ may depend on $f$.

For each system $(A, B)$, we associate the self-adjoint positive operators

$$
W(t, s)=\int_{s}^{t} U(s, \tau) B(\tau) B(\tau)^{*} U(s, \tau)^{*} d \tau
$$

for $t \geq s \geq 0$.

Recall (see [5], [8]) that a bounded linear control system $(A, B)$ is uniformly

Received by the editors Feb. 28, 1977 in revised form. 
completely controllable (and we write u.c.c.) iff there exist $\delta>0$ and $m>0$ such that

$$
W(s+\delta, s) \geq m \cdot I \text { for all } s \geq 0
$$

(where $I$ denotes the identity operator).

The B.I.B.O. stability of bounded finite dimensional systems is characterized by the following:

THEOREM 1.1. The bounded system $(A, B)$ is zero-state B.I.B.O. stable if and only if there exists a number $M>0$ such that

$$
\int_{t_{0}}^{t}\|U(t, s) B(s)\| d s \leq M
$$

for every $t \geq t_{0} \geq 0$.

For the proof see [2] pp. 385-386 or [3] pp. 194-195. By Theorem 1.1 and a Theorem of Silverman and Anderson ([5] Theorem 3) we have

THEOREM 1.2. If $(A, B)$ is bounded and uniformly completely controllable then it is zero state B.I.B.O. stable if and only if the equation $(A)$ is exponentially stable.

This paper contains two major results. The first one is to prove that the Theorem 1.1 is not true in the case of infinite dimensional systems. This proves that the use of the finite dimensional method for the proof of the Theorem 1.2 (see [5]) is not applicable in infinite dimensional case. The second is to introduce a new concept of input-output stability $\left(\left(L_{a}^{p}, L_{b}^{q}\right)\right.$ stability) and to give a large class of linear systems $(A, B)$ for which the $\left(L_{a}^{p}, L_{b}^{q}\right)$ stability implies the exponential stability of $(A)$.

2. Definitions, notations, and preliminary results. Let $X$ be a separable Hilbert space and consider the time-varying linear system

$$
\dot{x}(t)=A(t) x(t)+B(t) f(t), \quad t \geq 0,
$$

where $A \in L_{\mathrm{loc}}^{1}\left(R_{+}, L(X)\right), B \in \tau\left(R_{+}, L(Y, X)\right), f \in F \subset L_{\mathrm{loc}}^{1}\left(R_{+}, Y\right)$ in which $R_{+}=[0, \infty), L(Y, X)$ - the Banach space of the bounded linear operators from the Banach space $Y$ to $X$ (particularly $L(X, X)=L(X)$ ), $L_{\text {loc }}^{1}\left(R_{+}, Z\right)$-the space of $Z$-valued functions $f$ defined almost everywhere on $R_{+}$, such that $f$ is strongly measurable and locally Bochner integrable and, finally $\tau\left(R_{+}, L(Y, X)\right)$ - the space of continuous operator valued functions from $R_{+}$to $L(Y, X)$.

By the above hypotheses, the solution of $(A, B)$ with initial data $x\left(t_{0}\right)=x_{0}$ is ([10])

$$
x\left(t, t_{0} ; x_{0} ; f\right)=U\left(t, t_{0}\right) x_{0}+\int_{t_{0}}^{t} U(t, s) B(s) f(s) d s
$$


where $U\left(t, t_{0}\right)$ is the solution of Cauchy problem

$$
\left\{\begin{array}{l}
\dot{X}(t)=A(t) X(t) \\
X\left(t_{0}\right)=I
\end{array}\right.
$$

As a preliminary, we prove the following lemma which gives a characterization of uniform complete controllability.

LEMMA 2.1. The system $(A, B)$ is u.c.c. if and only if there exist $\delta>0$ and $k>0$ such that for every $x \in X$ and for any time $s \geq 0$ there exists an input $f$ such that

(i) $\|f(t)\| \leq k \cdot\|x\|$ for $t \in[s, s+\delta]$ and

(ii) $x=\int_{s}^{s+\delta} U(s, \tau) B(\tau) f(\tau) d \tau$.

Proof. If $(A, B)$ is u.c.c. then $W(s+\delta, s)$ are invertible operators and

$$
\left\|W(s+\delta, s)^{-1}\right\| \leq 1 / m \text { for every } s \geq 0 .
$$

Let $x \in X$ and $s \geq 0$. Define

$$
f(t)=\left\{\begin{array}{ccc}
-B(t)^{*} U(s, t)^{*} W(s+\delta, s)^{-1} x, & \text { if } & t \in[s, s+\delta] \\
0, & \text { if } t \notin[s, s+\delta]
\end{array}\right.
$$

It is clear that

$$
\|f(t)\| \leq k \cdot\|x\|, \text { where } k=K e^{K \delta} / m
$$

and

$$
x(s+\delta, s ; x ; f)=U(s+\delta, s)\left(x-W(s+\delta, s) W(s+\delta, s)^{-1} x\right)=0
$$

which implies that

$$
x=\int_{s}^{s+\delta} U(s, \tau) B(\tau) f(\tau) d \tau .
$$

The converse implication will be established by contradiction. If $(A, B)$ is not u.c.c. then for each $\delta>0$ and for any $\varepsilon>0$ there exists a vector $x_{0} \in X$ with $\left\|x_{0}\right\|=1$ such that

$$
\left\langle W\left(s_{0}+\delta, s_{0}\right) x_{0}, x_{0}\right\rangle=\int_{s_{0}}^{s_{0}+\delta}\left\|B(\tau)^{*} U\left(s_{0}, \tau\right)^{*} x_{0}\right\|^{2} d \tau<\varepsilon
$$

for some $s_{0} \geq 0$.

Assume that (i) and (ii) hold. Then for $x_{0}$ there exists an input $f_{0}$ such that $\left\|f_{0}(t)\right\| \leq k_{0}$ for $t \in\left[s_{0}, s_{0}+\delta\right]$ and

$$
x_{0}=\int_{s_{0}}^{s_{0}+\delta} U\left(s_{0}, \tau\right) B(\tau) f_{0}(\tau) d \tau
$$


By the Schwarz inequality it follows

$$
\begin{aligned}
1=\left\|x_{0}\right\|^{2} & =\int_{s_{0}}^{s_{0}+\delta}\left\langle f_{0}(t), B(t)^{*} U\left(s_{0}, t\right)^{*} x_{0}\right\rangle d t \\
& \leq\left(\int_{s_{0}}^{s_{0}+\delta} f_{0}(t) \|^{2} d t\right)^{1 / 2} \cdot\left(\int_{s_{0}}^{s_{0}+\delta}\left\|B(t)^{*} U\left(s_{0}, t\right)^{*} x_{0}\right\|^{2} d t\right)^{1 / 2} \\
& \leq \sqrt{ }(\delta \cdot \varepsilon) \cdot k_{0},
\end{aligned}
$$

which is a contradiction since $\varepsilon$ can be found arbitrarily small.

The following result can be found in Ref. [10].

Lemma 2.2. The equation $(A)$ is exponentially stable if and only if there exists a constant $N>0$ such that

$$
\int_{t_{0}}^{\infty}\left\|U\left(t, t_{0}\right) x\right\|^{q} d t \leq N, \quad(1 \leq q<\infty)
$$

for all $t_{0} \geq 0$ and for all $x \in X$ with $\|x\|=1$.

Let $a \geq 0$ and $1 \leq p \leq \infty . L_{a}^{p}(X)$ shall denote the Banach space of all $X$-valued, strongly measurable functions defined a.e. on $R_{+}$such that

$$
\begin{gathered}
\|f\|_{(a, p)}=\int_{R_{+}}\|f(s)\|^{p} \cdot e^{\text {pas }} d s<\infty \quad \text { if } p<\infty \\
\|f\|_{(a, \infty)}=\underset{s \geq 0}{\operatorname{ess} \sup }\|f(s)\| e^{a s}<\infty \quad \text { if } \quad p=\infty .
\end{gathered}
$$

(Particularly $L_{0}^{p}(X)=L^{p}(X)$ ).

3. EXAMPLE. The following example shows that, in contrast to the case where $X$ is finite dimensional, zero state B.I.B.O. stability does not imply the inequality (1.2).

Let $l^{2}$ be the Hilbert space of all real sequences $x=\left(x_{0}, x_{1}, x_{2}, \ldots, x_{n}, \ldots\right)$ such that $\sum_{n=0}^{\infty}\left|x_{n}^{2}\right|<\infty$.

On $l^{2}$ we define the bounded linear operator $B(t)$ defined by

$$
B(t) x=\frac{1}{[t]+1}\left(0,0, \ldots, 0, x_{[t]}, 0, \ldots\right)
$$

where $[t]$ denotes the integral part of $t$.

It is not difficult to verify that

for every $t \geq 0$.

$$
\|B(t)\|=\frac{1}{[t]+1} \leq 1
$$

On $l^{2}$ we will consider the control system $(0, B)$. Since

$$
\int_{0}^{\infty}\|U(t, s) B(s)\| d s=\int_{0}^{\infty}\|B(s)\| d s=\int_{0}^{\infty} \frac{1}{1+[s]} d s=\infty
$$

it follows that the inequality (1.2) is not true for the system $(0, B)$. 
Let $t \geq t_{0} \geq 0$ and let $f$ be an input such that $\|f(s)\| \leq M$ for every $s \geq t_{0}$.

Then there exists two positive integer numbers $m$ and $n$ such that $m<t_{0} \leq$ $m+1$ and $n<t \leq n+1$.

Since $\|f(s)\| \leq M$ it follows that

$$
\left|\int_{k-1}^{k} f_{k-1}(s) d s\right| \leq M,
$$

for every $k \geq m+1$, where $f(s)=\left(f_{0}(s), f_{1}(s), \ldots, f_{k}(s), \ldots\right)$.

By virtue of definition of norm on $l^{2}$ and by the Cauchy-Schwartz inequality we obtain:

$$
\begin{aligned}
\left\|x\left(t, t_{0} ; 0 ; f\right)\right\| & =\left\|\int_{t_{0}}^{t} U(t, s) B(s) f(s) d s\right\|=\| \frac{1}{m+1} \int_{t_{0}}^{m+1}\left(0,0, \ldots, f_{m}(s), \ldots\right) d s \\
& +\frac{1}{m+2} \int_{m+1}^{m+2}\left(0,0, \ldots, f_{m+1}(s), \ldots\right) d s+\ldots \\
& +\frac{1}{n+1} \int_{n+1}^{t}\left(0,0, \ldots, f_{n}(s), \ldots\right) d s \| \\
& =\|\left(0, \ldots, 0, \frac{1}{m+1} \int_{t_{0}}^{m+1} f_{m}(s) d s, \frac{1}{m+2}\right. \\
& \left.\times \int_{m+1}^{m+2} f_{m+1}(s) d s, \ldots, \frac{1}{n+1} \int_{n}^{t} f_{n}(s) d s, 0, \ldots\right) \| \\
& =\left[\sum_{j=m+2}^{n} \frac{1}{j^{2}}\left(\int_{j-1}^{j} f_{j-1}(s) d s\right)^{2}+\frac{1}{(m+1)^{2}}\left(\int_{t}^{m+1} f_{m}(s) d s\right)^{2}\right. \\
& \left.+\frac{1}{(n+1)^{2}}\left(\int_{n}^{t} f_{n}(s) d s\right)^{2}\right]^{1 / 2} \\
& \leq M\left(\sum_{j=m+1}^{n+1} \frac{1}{j^{2}}\right)^{1 / 2} \leq M \cdot\left(\sum_{j=1}^{\infty} \frac{1}{j^{2}}\right)^{1 / 2}=M \cdot \frac{\pi}{\sqrt{ } 6}<\infty
\end{aligned}
$$

and hence the system $(0, B)$ is zero state B.I.B.O. stable. Hence the Theorem 1.1 is false in the infinite dimensional case.

4. $\left(L_{a}^{p}, L_{b}^{q}\right)$ stability. Let $a, b \geq 0$ and $1 \leq p, q \leq \infty$.

Definition 4.1. The linear control system is said to be $\left(L_{a}^{p}, L_{b}^{q}\right)$ stable iff for every $f \in L_{a}^{p}(Y)$ the output

$$
x(t, 0 ; 0 ; f)=\int_{0}^{t} U(t, s) B(s) f(s) d s \text { is in } L_{b}^{q}(X) .
$$

LEMMA 4.1. If $(A, B)$ is $\left(L_{a}^{p}, L_{b}^{q}\right)$ stable then the operator $\Lambda: L_{a}^{p}(X) \rightarrow L_{b}^{q}(X)$ 
defined by

$$
(\Lambda f)(t)=\int_{0}^{t} U(t, s) B(s) f(s) d s
$$

is a bounded linear operator.

Proof. Let $f_{n} \rightarrow f$ in $L_{a}^{p}(X)$ and $\Lambda f_{n} \rightarrow g$ in $L_{b}^{q}(X)$.

Hence $h_{n} \rightarrow h$ in $L^{p}(X)$, where $h_{n}(s)=f_{n}(s) e^{a s}$ and $h(s)=f(s) e^{a s}$.

Since we are dealing with $L^{p}$ spaces, we can find a subsequence $h_{n_{k}}$ such that $h_{n_{k}}(t) \rightarrow h(t)$ a.e. on $R_{+}$(and hence $f_{n_{k}}(t) \rightarrow f(t)$ a.e. on $\left.R_{+}\right)$and

$$
\int_{0}^{t} U(t, s) B(s) f_{n_{k}}(s) d s \rightarrow g(t) \text { a.e. on } R_{+} .
$$

Because $U(t, s) B(s)$ is strongly continuous on $X$, this means that

$$
\left(\Lambda f_{n_{k}}\right)(t)=\int_{0}^{t} U(t, s) B(s) f_{n_{k}}(s) d s \rightarrow \int_{0}^{t} U(t, s) B(s) f(s) d s
$$

for all $t \in R_{+}$. Hence, $g(t)=\int_{0}^{t} U(t, s) B(s) f(s) d s$ a.e. on $R_{+}$.

The closed graph theorem ([12]) shows that $\Lambda$ is continuous.

Corollary 4.2. If $(A, B)$ is $\left(L_{a}^{p}, L_{b}^{\infty}\right)$ stable then there exists $M>0$ such that

$$
e^{b t} \cdot\left\|\int_{0}^{t} U(t, s) B(s) f(s) d s\right\| \leq M \cdot\|f\|_{(p, a)}
$$

THEOREM 4.3. If the system $(A, B)$ is u.c.c. and $\left(L_{a}^{p}, L_{b}^{\infty}\right)$ stable then there exists $N>0$ such that

$$
\left\|U\left(t, t_{0}\right)\right\| \leq N \cdot e^{a t_{0}} e^{-b t}
$$

for all $t \geq t_{0} \geq 0$.

Proof. By u.c.c. of $(A, B)$ we have that there exist $\delta>0$ and $m>0$ such that $W(s+\delta, s)$ are invertible operators and

$$
\left\|W(s+\delta, s)^{-1}\right\| \leq 1 / m, \text { for all } s \geq 0 .
$$

Let $t \geq \delta$ and let $n$ be a positive integer such that $t \geq(n+1) \delta$. Let $f_{n}$ be the control function given by

$$
f_{n}(s)=\left\{\begin{array}{ccc}
K_{1} B(s)^{*} U(n \delta, s)^{*} x, & \text { if } & s \in[n \delta,(n+1) \delta] \\
0, & \text { if } & s \notin[n \delta,(n+1) \delta],
\end{array}\right.
$$

where $x \in X$ with $\|x\|=1$ and $K_{1}=1 /\left(K \cdot e^{K \delta} \cdot \sqrt[p]{\delta} \delta\right.$.

Clearly $f_{n} \in L_{a}^{p}(Y)$ and by

$$
\|U(n \delta, s)\| \leq e \int_{n \delta}^{s}\|A(s)\| d s \leq e^{K \delta}, \quad \text { for } \quad s \leq(n+1) \delta
$$


and by (2.1) we then obtain $\left\|f_{n}\right\|_{(p, a)} \leq e^{(n+1) a \delta}$. Using the Corollary 4.2. it follows:

and hence

$$
\begin{aligned}
M e^{(n+1) a} e^{-b t} & \geq\left\|\int_{0}^{t} U(t, s) B(s) f_{n}(s) d s\right\| \\
& =K_{1}\|U(t, n \delta) W((n+1) \delta, n \delta) x\|
\end{aligned}
$$

$$
\|U(t, n \delta) W((n+1) \delta, n \delta)\| \leq \frac{M}{K_{1}} \cdot e^{(n+1) a \delta} \cdot e^{-b t}
$$

for every $t \geq(n+1) \delta$. By u.c.c. of $(A, B)$ we obtain

$$
\begin{aligned}
\|U(t, n \delta)\| & \leq\|U(t, n \delta) W((n+1) \delta, n \delta)\| \cdot\left\|W((n+1) \delta, n \delta)^{-1}\right\| \\
& \leq M_{1} e^{(n+1) a \delta} \cdot e^{-b t}
\end{aligned}
$$

for $t \geq(n+1) \delta$, where $M_{1}=M / m \cdot K_{1}$.

(i) Let $t \geq \delta, t_{0} \in[\delta, t]$ and let $n$ be the positive integer such that $(n+1) \delta \leq$ $t_{0}<(n+2) \delta$. Then

$$
\begin{aligned}
\left\|U\left(t, t_{0}\right)\right\| & \leq\|U(t, n \delta)\| \cdot\left\|U\left(n \delta, t_{0}\right)\right\| \leq M_{1} e^{(n+1) a \delta} \cdot e^{-b t} \cdot e^{K\left(t_{0}-n \delta\right)} \\
& \leq M_{1} e^{2 K \delta} e^{a t_{0}} e^{-b t}=M_{2} e^{a t_{0}} e^{-b t} .
\end{aligned}
$$

(ii) If $t \geq \delta \geq t_{0} \geq 0$ then

$$
\begin{aligned}
\left\|U\left(t, t_{0}\right)\right\| & \leq\|U(t, \delta)\| \cdot\left\|U\left(\delta, t_{0}\right)\right\| \leq M_{2} e^{a \delta} \cdot e^{-b t} \cdot e^{K\left(\delta-t_{0}\right)} \\
& =M_{3} e^{a t_{0}} e^{-b t}, \text { where } M_{3}=e^{\delta(K+a)} .
\end{aligned}
$$

(iii) Let $t_{0} \geq 0$ and $t \geq t_{0}$.

If $m_{4}=\sup \left\{\left\|U\left(t, t_{0}\right)\right\| e^{-a t_{0}} \cdot e^{b t}, 0 \leq t_{0} \leq t \leq \delta\right\}$ and $N=\max \left\{M_{2}, M_{3}, M_{4}\right\}$ then we have

$$
\left\|U\left(t, t_{0}\right)\right\| \leq N e^{-a t_{0}} \cdot e^{b t}
$$

for all $t_{0} \geq 0$ and $t \geq t_{0}$.

COROLlaRy 4.4. If the system $(A, B)$ is u.c.c. and $\left(L_{a}^{p}, L_{b}^{\infty}\right)$ stable (where $1 \leq p \leq \infty$ and $0 \leq a<b$ or $0<a \leq b)$ then the equation $(A)$ is exponentially stable.

THEOREM 4.5. If the system $(A, B)$ is u.c.c. and $\left(L_{0}^{p}, L_{b}^{q}\right)$ stable (where $b \geq 0$, $1 \leq p \leq \infty$, and $1 \leq q<\infty)$ then the equation $(A)$ is exponentially stable.

Proof. Let $x \in X$ with $\|x\|=1$. From Lemma 2.1. it follows that there exist $\delta>0$ and $k>0$ such that for all $t_{0} \geq 0$, there exists an input $f$ which satisfies $\|f(s)\| \leq k$ for $s \in\left[t_{0}, t_{0}+\delta\right]$ and

$$
x=\int_{t_{0}}^{t_{0}+\delta} U\left(t_{0}, s\right) B(s) f(s) d s .
$$


Let

$$
g(s)=\left\{\begin{array}{rrr}
f(s), & \text { if } & s \in\left[t_{0}, t_{0}+\delta\right] \\
0, & \text { if } & s \notin\left[t_{0}, t_{0}+\delta\right]
\end{array}\right.
$$

Clearly $g \in L_{a}^{p}(Y)$ and

$$
(\Lambda g)(t)=x(t, 0 ; 0 ; g)=\left\{\begin{array}{cl}
0, & \text { if } t \leq t_{0} \\
\int_{t_{0}}^{t} U(t, s) B(s) f(s) d s, & \text { if } t_{0} \leq t \leq t_{0}+\delta \\
\int_{t_{0}}^{t_{0}+\delta} U(t, s) B(s) f(s) d s=U\left(t, t_{0}\right) x, & \text { if } t>t_{0}+\delta .
\end{array}\right.
$$

Using the $\left(L_{0}^{p}, L_{b}^{q}\right)$ stability we have

$$
\begin{aligned}
\int_{t_{0}}^{\infty}\left\|U\left(t, t_{0}\right) x\right\|^{q} e^{q b t} d s & \leq \int_{0}^{\infty}\|(\Lambda g)(t)\|^{q} e^{q b t}=\left\|\Lambda_{g}\right\|_{(q, b)}^{q} \\
& \leq M \cdot\|g\|_{(p, 0)}^{q} \leq(M \cdot k \cdot \delta)^{q}
\end{aligned}
$$

and hence

$$
\begin{aligned}
\int_{t_{0}}^{\infty}\left\|U\left(t, t_{0}\right) x\right\|^{q} d t & \leq \int_{t_{0}}^{t_{0}+\delta}\left\|U\left(t, t_{0}\right) x\right\|^{q} d t+\int_{t_{0}+\delta}^{\infty}\left\|U\left(t, t_{0}\right) x\right\|^{q} e^{q b t} d t \\
& \leq N=e^{K \delta q}+(M k \delta)^{q},
\end{aligned}
$$

for every $t_{0} \geq 0$ and for all $x \in X$ with $\|x\|=1$. By Lemma 2.2 it follows that $(A)$ is exponentially stable.

\section{REFERENCES}

1. O. Perron, Die Stabilitätsfrage der Differentialgleichungen, Math. Z., 32 (1930), 703-728.

2. L. A. Zadeh and C. A. Desoer, Linear System Theory, McGraw-Hill, New York, 1963.

3. R. Brockett, Finite Dimensional Linear Systems, John Wiley, New York, 1970.

4. L. Haines and L. M. Silverman, Internal and external stability of linear systems, J. Math. Anal. Appl. 21 (1968), 277-286.

5. L. M. Silverman and B. D. O. Anderson, Controllability, observability and stability of linear systems, Siam. J. Control, 6 (1968), 121-130.

6. M. Megan, On the stability of linear controllable systems in Hilbert spaces, Proc. Conf. Funct. Equations, Iassy, 1973.

7. M. Megan, Stabilitatea sistemelor liniare cu control în spaţii Hilbert, Seminarul de Ecuaţii Functionale Timişoara, 14 (1973).

8. M. Megan and V. Hiriş, Controlabilitatea, Stabilitatea şi Optimizarea Sistemelor Liniare în Spaţii Hilbert, Universitatea din Timişoara, 1975.

9. M. Reghiş, Asupra stabilitaţii neuniforme în spaţii generale Lucrările ştiinţ̧fice ale Inst. Pedag. Timişoara (1960), 153-169.

10. J. L. Daletsky and M. G. Krein, Stability of Solutions of Differential Equations in Banach Spaces, Nauka, Moscow, 1970, published in English by the American Mathematical Society. 
11. M. Megan and V. Hiriş, On the space of linear controllable systems in Hilbert spaces, Glasnik Matematicki 10 (1975), 161-167.

12. K. Yosida, Functional Analysis, Springer Verlag, New York, 1967.

\section{UNIVERSITY OF TIMISSOARA}

DEPARTMENT OF MATHEMATICS

BUL. V. PÂRVAN NR. 4

1900-TIMIŞOARA

R. S. ROMANIA 